\title{
Nasal symptoms following endoscopic transsphenoidal pituitary surgery: assessment using the General Nasal Patient Inventory
}

\author{
Yi Yuen Wang, M.D., ${ }^{1}$ Vinothan Srirathan, M.B.Ch.B., ${ }^{1}$ Erica Tirr, M.B.Ch.B., ${ }^{1}$ \\ Tara Kearney, M.D., ${ }^{2}$ and Kanna K. Gnanalingham, Ph.D. ${ }^{1}$ \\ Departments of ${ }^{I}$ Neurosurgery and ${ }^{2}$ Endocrinology, Greater Manchester Neuroscience Centre, Salford Royal \\ Foundation Trust, Salford, United Kingdom
}

\begin{abstract}
Object. The endoscopic approach for pituitary tumors is a recent innovation and is said to reduce the nasal trauma associated with transnasal transsphenoidal surgery. The authors assessed the temporal changes in the rhinological symptoms following endoscopic transsphenoidal surgery for pituitary lesions, using the General Nasal Patient Inventory (GNPI).

Methods. The GNPI was administered to 88 consecutive patients undergoing endoscopic transsphenoidal surgery at 3 time points (presurgery, 3-6 months postsurgery, and at final follow-up). The total GNPI score and the scores for the individual GNPI questions were calculated and differences between groups were assessed once before surgery, several months after surgery, and at final follow-up.

Results. Of a maximum possible score of 135, the mean GNPI score at 3-6 months postsurgery was only 12.9 \pm 12 and was not significantly different from the preoperative score $(10.4 \pm 13)$ or final follow-up score $(10.3 \pm 10)$. Patients with functioning tumors had higher GNPI scores than those with nonfunctioning tumors for each of these time points $(\mathrm{p}<0.05)$. Individually, a mild increase in symptom severity was seen for symptoms attributable to the nasal trauma of surgery, with partial recovery (nasal sores and bleeding) or complete recovery (nasal blockage, painful sinuses, and unpleasant nasal smell) by final follow-up ( $\mathrm{p}<0.05)$. Progressive improvements in symptom severity were seen for symptoms more attributable to tumor mass preoperatively (for example, headaches and painkiller use [p $<0.05])$. In total, by final follow-up 8 patients $(9 \%)$ required further treatment or advice for ongoing nasal symptoms.

Conclusions. Endoscopic transsphenoidal surgery is a well-tolerated minimally invasive procedure for pituitary fossa lesions. Overall patient-assessed nasal symptoms do not change, but some individual symptoms may show a mild worsening or overall improvement. (DOI: 10.3171/2011.1.FOCUS10319)
\end{abstract}

\section{KeY Words - endoscopic transsphenoidal surgery - pituitary tumor • nasal symptoms}

$\mathrm{T}$ HE endoscopic approach for pituitary tumors is a recent innovation, and there are now several reports of patients in whom the endoscopic transnasal transsphenoidal route was used, with claims of benefits for the surgeon and patient. ${ }^{2,13,17}$ Apart from the improved visualization provided by both a wider field of view and focused illumination, the endoscopic approach is also claimed to be associated with fewer nasal complications..$^{2,6,19}$

Outcome measures following transsphenoidal surgery have traditionally included the following: assessment of the reversal of visual field deficits, extent of MR imaging-documented tumor resection, and the remission rates for functioning adenomas. These measures focus

Abbreviation used in this paper: GNPI = General Nasal Patient Inventory. primarily on the clinician's perspective of the patient's illness but may not take into account the patient's concerns with his or her own health. Patient-based questionnaires are increasingly being validated for use as outcome measures, providing individualized evaluation of the success of the given therapy.1,11

The GNPI is a patient-oriented, general nasal symptom questionnaire that has been validated in large numbers of patients with rhinological concerns. ${ }^{7}$ It represents a comprehensive 45 -item questionnaire, encompassing a vast array of nasal complaints perceived as important by patients (Table 1). It is a sensitive tool in assessing preand postoperative nasal symptoms following any nasal intervention. ${ }^{3}$ In this study, we administered the GNPI to patients undergoing endoscopic transsphenoidal pituitary surgery, and we report on the temporal changes in patientperceived rhinological symptoms. 


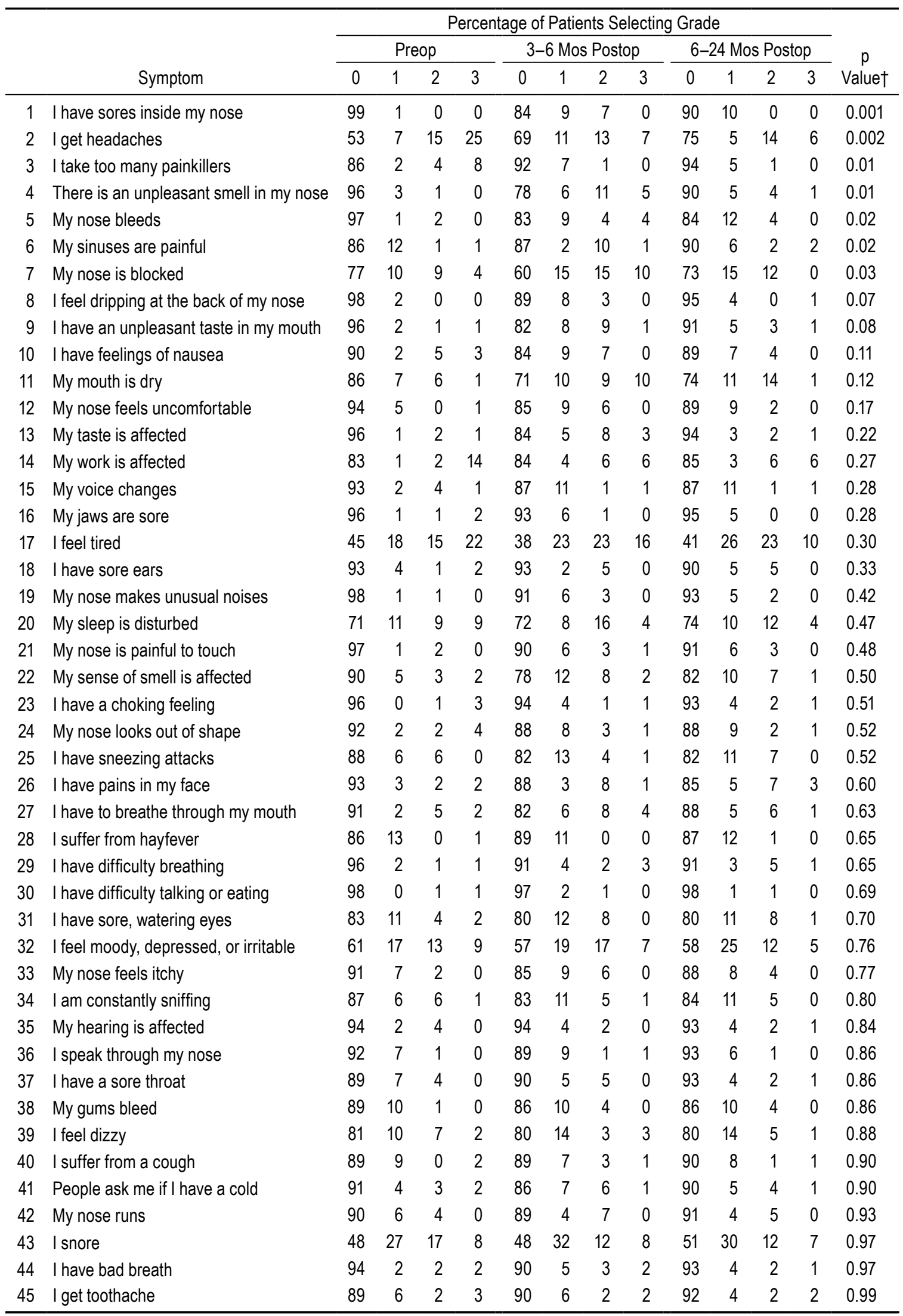

* Grades are defined as the following: $0=$ no symptom; $1=$ mild symptom; 2 = moderate symptom; 3 = severe symptom.

† Pearson chi-square test. 


\section{Patient-assessed nasal symptoms in endoscopic pituitary surgery}

\section{Methods}

One hundred consecutive patients undergoing endoscopic transsphenoidal pituitary surgery were asked to participate after informed consent. For each patient, the clinical presentation, endocrine profile, visual field changes, MR imaging findings, operative details, complications, and tumor pathology were noted. Any history of nasal trauma or intervention was recorded.

\section{Operative Technique}

The endoscopic transnasal transsphenoidal approach we used has been described in detail. ${ }^{15}$ Specifically, nasal preparation was performed with topical application of preoperative decongestants (cophenylcaine, Aurum Ltd.) into both nostrils. No antiseptic wash or injection of vasoconstrictors was used. A single-nostril approach is generally preferred with the side of access chosen following visual identification of the more capacious nasal passage or by the laterality of the lesion. The middle turbinate is gently deflected laterally to allow access. Turbinectomy (partial or total) is not undertaken. The posterior nasal mucosa overlying the anterior sphenoid wall and vomer is cauterized before opening the sphenoid sinus. The sphenoid sinus mucosa is usually retracted laterally using cotton patties. In the event of CSF leakage, a graded operative repair was undertaken using combinations of hemostatic gelatin sponge (Spongistan, Ethicon), dural substitute (Durafoam, Codman), periumbilical fat graft, and a dural sealant (Duraseal, Confluent Surgical). ${ }^{18}$ Vascularized mucosal flaps are not used as part of our repair. ${ }^{18}$ Patients undergoing extended transsphenoidal procedures were not included in this study.

Closure following tumor removal involves inspection of both nasal cavities to identify significant mucosal bleeding points that are controlled with diathermy. The deflected middle turbinate is medialized. Nasal packing is not routinely employed postoperatively, and the topical application of a nasal decongestant (xylometazoline $0.1 \%$ spray, Novartis) is prescribed for 7 days only. Postoperatively patients do not receive any other routine nasal treatment such as irrigation or debridement unless one is clinically deemed necessary.

\section{Nasal Symptoms}

The patients were asked to complete the GNPI questionnaire to assess nasal symptoms at 3 time points: preoperatively, 3-6 months postoperatively, and at final neurosurgical follow-up (Table 1). For each of the 45 items of the GNPI questionnaire the patient had to choose among 4 numerical answers $(0=$ not present, $1=$ mild, $2=\bmod -$ erate, and $3=$ severe) $($ Table 1$)$. Total scores $(0-135)$ were graded per patient and per item, allowing assessment of global as well as specific changes in nasal symptoms over time.

\section{Statistical Analysis}

All data were entered and analyzed using the SPSS statistical package (Statistical Programs for the Social Sciences). Differences between groups were assessed using an ANOVA and post hoc Bonferroni tests for para- metric data and using the chi-square tests for nonparametric data.

\section{Results}

\section{Demographic and Pathological Data}

Of the 100 patients approached to participate in the study, 88 patients provided complete data, and the data obtained in these 88 patients were included in the present study. The mean age $( \pm$ SD) of the patients was $51 \pm$ 15 years (range 18-77 years), with a male preponderance (male/female ratio 1.4:1). The mean follow-up duration was $13 \pm 4$ months (range 6-23 months). Pituitary macroadenomas were present in 74 patients $(84 \%)$, and the remaining tumors were as follows: 8 microadenomas, 5 cystic lesions (4 craniopharyngiomas and 1 Rathke cleft cyst), and 1 clival lesion. Surgery was performed for nonfunctioning pituitary adenomas in 54 cases $(61 \%)$, hormone-secreting adenomas in 25 cases (28\% [acromegaly in 14 , Cushing disease in 5, prolactinoma in 4, and thyroid-stimulating hormone-secreting in 1]), and miscellaneous in 9 (10\% [4 craniopharyngioma, 2 Rathke cleft cyst, and 4 apoplexy]). None of the patients underwent an extended transsphenoidal procedure.

Five patients underwent a redo transsphenoidal procedure, with the first procedure being a microscopic transsphenoidal operation performed by another surgeon in all cases. Of these patients, 2 had undergone radiotherapy following their original procedure. Intraoperative CSF leaks were seen in 22 patients (25\%), with all leaks intraoperatively repaired using a combination of collagen sponge, hydrogel sealant, and occasionally periumbilical fat grafts when large arachnoid defects were noted.

\section{General Nasal Patient Inventory Scores}

Overall, there was no significant change in the total GNPI scores at 3-6 months or at final follow-up compared with the preoperative scores $(\mathrm{p}=0.3$, ANOVA) (Fig. 1). Functioning tumors were associated with higher GNPI scores than nonfunctioning tumors for each of these time points $(\mathrm{p}<0.01$, ANOVA) (Fig. 2). There was no significant difference between different types of functioning tumors. Other factors such as tumor size, intraoperative CSF leakage, and the nostril of approach did not affect the GNPI score (data not shown).

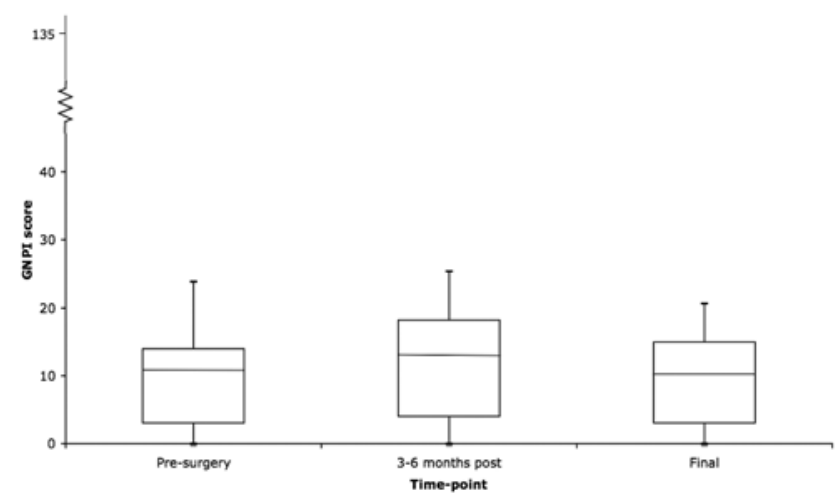

FIG. 1. Changes in total GNPI scores over time (mean and quartile values $(p=0.3$, ANOVA). . Final $=$ final follow-up; post $=$ postoperatively . 


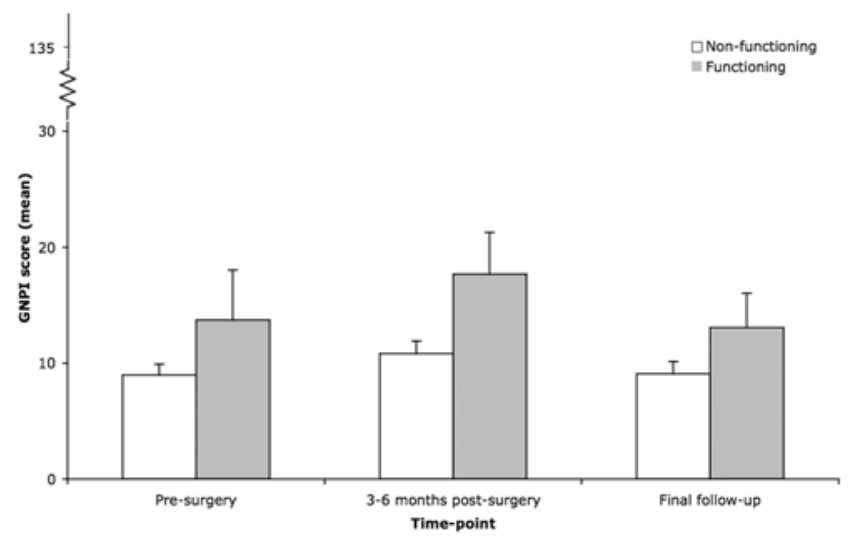

FIG. 2. Mean GNPI scores for functioning and nonfunctioning tumors over time. Functioning tumors were associated with a significantly higher GNPI score than nonfunctioning tumors at all time points $(p<$ 0.01 , ANOVA).

When considering the individual symptoms within the GNPI, several patterns of symptom progression were apparent over the study period. A transient worsening and partial reversal to preoperative levels were seen in response to questions related to nasal sores $(\mathrm{p}<0.01)$ and nasal bleeds ( $\mathrm{p}<0.05$, chi-square test) (Fig. 3C and D). A transient worsening and complete reversal to preoperative levels were seen in response to questions related to nasal blockage $(\mathrm{p}<0.05)$, unpleasant nasal smells $(\mathrm{p}<$ 0.01 ), and painful sinuses ( $\mathrm{p}<0.05$, chi-square test) (Fig. $3 \mathrm{E}$ and $\mathrm{G})$. Progressive improvement in symptom severity was seen in response to questions related to headaches
( $\mathrm{p}<0.01)$ and painkiller usage $(\mathrm{p}<0.01$, chi-square test) (Fig. 3A and B).

By the final follow-up, 8 patients (9\%) also required further clinical review for ongoing nasal symptoms such as nasal discharge/crusting (2 cases), blockage (2 cases), and intermittent bleeds (4 cases). These patients were managed with advice (4 cases), a course of steroid nasal sprays ( 2 cases), and cautery ( 2 cases).

\section{Discussion}

Endoscopic transsphenoidal surgery is increasingly accepted as the first-line approach for sella region pathological entities. ${ }^{3-5,8,14}$ Among the many purported advantages of endoscopic transsphenoidal surgery over microscopic approaches, one claim is a decrease in nasal trauma. ${ }^{12}$ In endoscopic approaches, the avoidance of a rigid nasal speculum can help minimize the trauma and distortion of the nasal turbinates and septum. To our knowledge, thus far there has not been a quantitative study investigating patients' nasal symptoms following endoscopic transsphenoidal surgery. We present our findings after administering the GNPI to assess each patient's perspective of his or her postoperative nasal symptoms.

The GNPI was developed specifically to assess nasal symptoms in patients rhinological conditions. ${ }^{7}$ This comprehensive 45-item inventory has been validated against the physician-derived 12-item Fairley nasal questionnaire. ${ }^{10}$ It assesses the "success" of surgery from the patient's perspective and has been shown to be more sensitive to change after intervention than other available mea-
Progressive Improvement

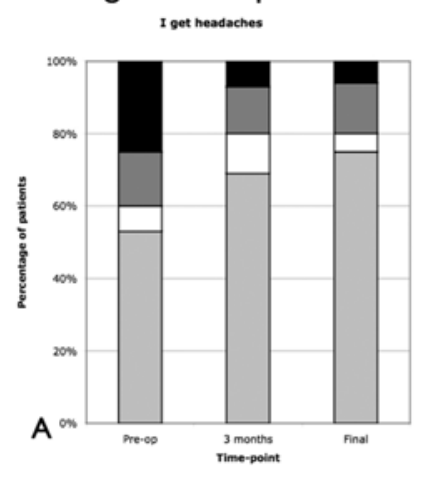

I take too many painkillers

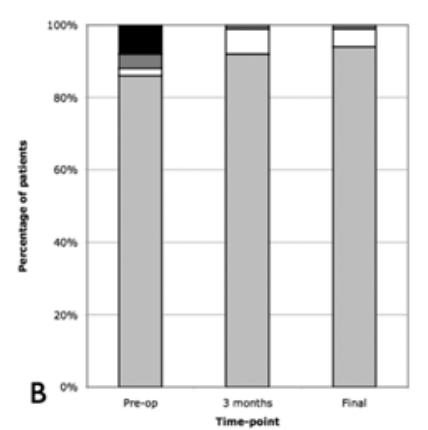

Transient Rise \& Partial Recovery
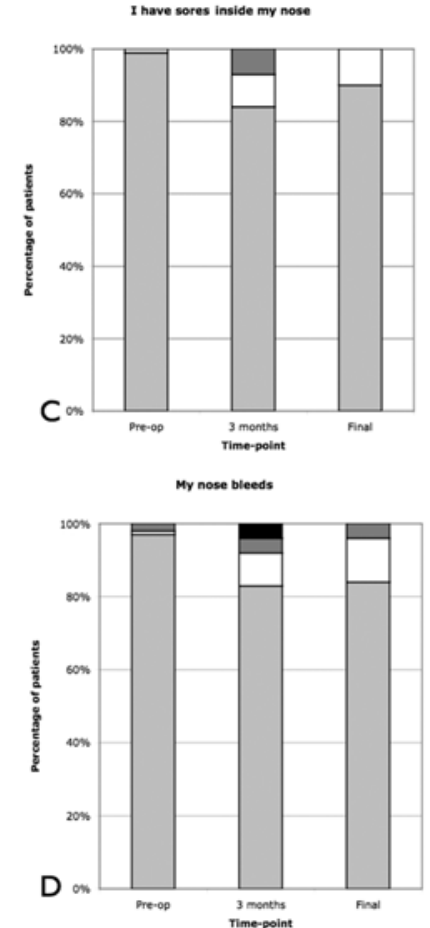

Transient Rise \& Complete Recovery
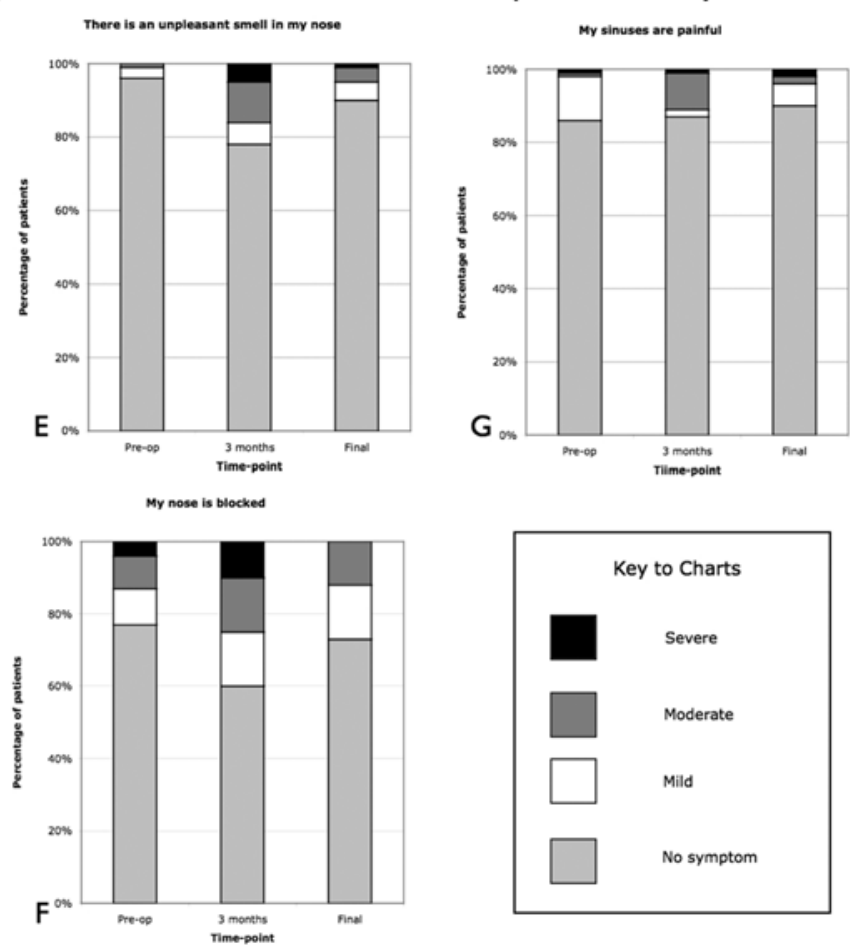

FIG. 3. Symptoms shown to be significantly changed following endoscopic transsphenoidal surgery. Some symptoms demonstrated a transient worsening followed by partial $(\mathbf{C}$ and $\mathbf{D})$ or complete $(\mathbf{E}-\mathbf{G})$ resolution $(p<0.05$, chi-square test). Other symptoms ( $A$ and $B$ ) showed a progressive improvement $(p<0.05$, chi-square test). 


\section{Patient-assessed nasal symptoms in endoscopic pituitary surgery}

sures. ${ }^{7}$ Although the GNPI was not specifically devised for endoscopic pituitary surgery, given the commonality of the surgical approach it seems logical to use it in this context also.

Although the GNPI has a maximum total score of 135, the highest mean score recorded after endoscopic transsphenoidal surgery was only 13 (range 0-74) at 3-6 months postoperatively (Fig. 1). This was also not significantly different from the preoperative scores and would suggest that endoscopic pituitary surgery is associated with minimal disturbance to the nasal passages in the long run. Functioning adenomas were associated with a higher GNPI score than nonfunctioning tumors at all time points (Fig. 2). This may in part be attributed to the propensity for some functioning tumors, in particular growth hormone-secreting tumors, to present with nasal and upper-airway symptoms.

When individual nasal symptoms were taken into consideration, several patterns of symptom progression were apparent over the study period. By 3-6 months postoperatively, a small rise in some symptoms attributable to the nasal trauma of surgery was noted (Fig. 3). These symptoms had partially recovered (nasal sores and nasal bleeding) or fully recovered (nasal blockage, unpleasant nasal smells, and sinus pain) by final follow-up. Endoscopic transsphenoidal surgery approaches require some coagulation and disruption of the posterior nasal mucosa, and, on the one hand, it is likely that the mild rise in symptom severity is attributable to this. On the other hand, there was a progressive improvement in other symptoms such as headaches and analgesic use (Fig. 3). This may be attributable to the mass effect and/or hormonal activity of the tumor. However, for the vast majority of symptoms assessed in the GNPI, there was no significant change in the severity, further confirming the minimal disturbances to the nasal passages caused by the endoscopic approach.

Furthermore, only $9 \%$ of patients required further clinical review and/or minor interventions for ongoing problems such as nasal discharge, blockage, or intermittent nasal bleeds. We acknowledge that in the absence of routine postoperative rhinological inspections it is impossible to know the true incidence of such nasal complications. However, our data suggest that the rate of such complications appears to be low and intervention is rarely required.

Previous studies reporting on nasal symptoms after pituitary surgery have been largely qualitative in nature and undertaken by retrospective review of case records. ${ }^{2,9,16,19}$ Moreover, the endoscopic approach is also claimed to be associated with fewer nasal complications compared with microscopic transsphenoidal approaches. ${ }^{2,19}$ Localized nasal complications such as obstruction and crusting have been reported in up to $38 \%$ of microscopic transsphenoidal surgeries..$^{9,16}$ Dusick et al. ${ }^{9}$ provided the only other quantitative assessment of the success of microscopic endonasal transsphenoidal surgery from the patients' perspective. They reported that by 3 months after microscopic transsphenoidal surgery, $67 \%-87 \%$ of patients had no rhinological complaints and concluded that rhinological recovery is rapid and relatively complete following microscopic endonasal transsphenoidal surgery. This is in keeping with the findings of the present study. It is difficult to compare the studies because of methodological differences, notably the use of 2 separate questionnaires.

\section{Conclusions}

Endoscopic transsphenoidal surgery is well tolerated for sella and parasellar lesions. Overall nasal symptoms do not change, but some individual symptoms may show mild worsening and/or improvement.

\section{Disclosure}

The authors report no conflict of interest concerning the materials or methods used in this study or the findings specified in this paper.

Author contributions to the study and manuscript preparation include the following. Conception and design: Gnanalingham. Acquisition of data: all authors. Analysis and interpretation of data: all authors. Drafting the article: Wang. Critically revising the article: Wang, Kearney, Gnanalingham. Reviewed final version of the manuscript and approved it for submission: Wang, Kearney, Gnanalingham. Statistical analysis: Wang, Gnanalingham. Study supervision: Gnanalingham.

\section{References}

1. Barcham LJ, Stephens SD: The use of an open-ended problems questionnaire in auditory rehabilitation. Br J Audiol 14: 49-54, 1980

2. Cappabianca P, Cavallo LM, Colao A, de Divitiis E: Surgical complications associated with the endoscopic endonasal transsphenoidal approach for pituitary adenomas. J Neurosurg 97:293-298, 2002

3. Cappabianca P, Cavallo LM, Esposito F, De Divitiis O, Messina A, De Divitiis E: Extended endoscopic endonasal approach to the midline skull base: the evolving role of transsphenoidal surgery. Adv Tech Stand Neurosurg 33:151-199, 2008

4. Cavallo LM, de Divitiis O, Aydin S, Messina A, Esposito F, Iaconetta G, et al: Extended endoscopic endonasal transsphenoidal approach to the suprasellar area: anatomic considerations-part 1. Neurosurgery 61 (3 Suppl):24-34, 2007

5. de Divitiis E, Cavallo LM, Cappabianca P, Esposito F: Extended endoscopic endonasal transsphenoidal approach for the removal of suprasellar tumors: Part 2. Neurosurgery 60:46-59, 2007

6. Dorward NL: Endocrine outcomes in endoscopic pituitary surgery: a literature review. Acta Neurochir (Wien) 152: 1275-1279, 2010

7. Douglas SA, Marshall AH, Walshaw D, Robson AK, Wilson JA: The development of a General Nasal Patient Inventory. Clin Otolaryngol Allied Sci 26:425-429, 2001

8. Dumont AS, Kanter AS, Jane JA Jr, Laws ER Jr: Extended transsphenoidal approach. Front Horm Res 34:29-45, 2006

9. Dusick JR, Esposito F, Mattozo CA, Chaloner C, McArthur DL, Kelly DF: Endonasal transsphenoidal surgery: the patient's perspective-survey results from 259 patients. Surg Neurol 65:332-342, 2006

10. Fairley JW, Yardley MP, Durham LH, Parker AJ: Reliability and validity of a nasal symptom questionnaire for use as an outcome measure in clinical research and audit of functional endoscopic sinus surgery. Clin Otolaryngol 18:436-437, 1993 (Abstract)

11. Gerszten PC: Outcomes research: a review. Neurosurgery 43: 1146-1156, 1998

12. Gondim JA, Almeida JP, de Albuquerque LA, Gomes E, Schops M, Ferraz T: Pure endoscopic transsphenoidal surgery 
for treatment of acromegaly: results of 67 cases treated in a pituitary center. Neurosurg Focus 29(4):E7, 2010

13. Jane JA Jr, Han J, Prevedello DM, Jagannathan J, Dumont AS, Laws ER Jr: Perspectives on endoscopic transsphenoidal surgery. Neurosurg Focus 19(6):E2, 2005

14. Kassam A, Carrau RL, Snyderman CH, Gardner P, Mintz A: Evolution of reconstructive techniques following endoscopic expanded endonasal approaches. Neurosurg Focus 19(1):E8, 2005

15. Leach P, Abou-Zeid AH, Kearney T, Davis J, Trainer PJ, Gnanalingham $\mathrm{KK}$ : Endoscopic transsphenoidal pituitary surgery: evidence of an operative learning curve. Neurosurgery 67:1205-1212, 2010

16. Petry C, Leães CG, Pereira-Lima JF, Gerhardt KD, Sant GD, Oliveira Mda C: Oronasal complications in patients after transsphenoidal hypophyseal surgery. Braz J Otorhinolaryngol 75:345-349, 2009
17. Powell M: Microscope and endoscopic pituitary surgery. Acta Neurochir (Wien) 151:723-728, 2009

18. Wang YY, Kearney T, Gnanalingham KK: Low-grade CSF leaks in endoscopic trans-sphenoidal pituitary surgery: efficacy of a simple and fully synthetic repair with a hydrogel sealant. Acta Neurochir (Wien) [epub ahead of print], 2010

19. White DR, Sonnenburg RE, Ewend MG, Senior BA: Safety of minimally invasive pituitary surgery (MIPS) compared with a traditional approach. Laryngoscope 114:1945-1948, 2004

Manuscript submitted December 15, 2010.

Accepted January 20, 2011.

Address correspondence to: Yi Yuen Wang, M.D., Department of Neurosurgery, Greater Manchester Neuroscience Centre, Salford Royal Foundation Trust, Stott Lane, Salford, M8 6HD, United Kingdom. email: yiyuen.wang@svhm.org.au. 